\title{
Pentoxifylline modulates LPS-induced hyperinflammation in monocytes of preterm infants in vitro
}

\author{
Simone S. Schüller ${ }^{1}$, Lukas Wisgrill ${ }^{1}$, Elisabeth Herndll ${ }^{1}$, Andreas Spittler ${ }^{2}$, Elisabeth Förster-Waldl ${ }^{1}$, Kambis Sadeghi ${ }^{1}$, \\ Boris W. Kramer ${ }^{3,4}$ and Angelika Berger ${ }^{1}$
}

BACKGROUND: Pentoxifylline (PTX), a methylxanthine derivate with immunomodulating properties, has been used as adjunctive treatment in severe neonatal sepsis. The aim of the study was to investigate the anti-inflammatory effects of PTX on Lipopolysaccharides (LPS)-stimulated monocytes of preterm neonates in vitro compared with monocytes of term infants and adult controls.

METHODS: Whole cord blood samples and control adult blood samples were incubated with LPS and PTX. The expression of surface markers, phagocytosis, cytokine secretion, and Toll-like receptor (TLR)4 signaling of monocytes were assessed by flow cytometry. Changes of TLR4-messenger RNA (mRNA) levels were confirmed by reverse-transcriptase PCR.

RESULTS: The expression of CD14, CD11b, CD64, CD71, and CD80 was downregulated by PTX in a dose-dependent manner; the greatest effect was observed on CD14 and CD11b in preterm infants. PTX markedly downregulated LPS-induced tumor necrosis factor-a, interleukin (IL)-1 $\beta$, and IL-6 levels in all age groups. Early IL-10 production was significantly downregulated by PTX in term and preterm neonates, while remaining unchanged in adults. Moreover, PTX downregulated TLR4 expression of monocytes on cellular and mRNA level, decreased signaling, and suppressed phagocytosis.

CONCLUSION: PTX downregulated TLR4 expression and signaling, thereby leading to strong anti-inflammatory properties in monocytes. Age-dependent differences were identified for CD14 and CD11b expression and IL-10 production.

$\mathbf{N}$ eonatal sepsis is associated with significant mortality in preterm infants (1). Understanding of the pathophysiologic processes surrounding neonatal sepsis and specifically the individual immunologic responses to neonatal sepsis is yet to be elucidated. Compared with adults, the neonatal immune system is compromised both in its humoral and cellular components. Levels of receptor expression, cytokine, and mediator kinetics differ significantly between neonates and adults (2). Therefore, therapies that target adult sepsis-specific deficits may have a differential effect in neonates.

Neonatal antigen-presenting cells (APCs) demonstrate an impaired production of Th1-polarizing cytokines and an impaired upregulation of co-stimulatory molecules to most Toll-like-receptor (TLR) agonists as well as an impaired TLR signaling (3-5). Monocytes present antigens by means of expression of HLA receptors and secrete cytokines to amplify the immune response (6). Multiple studies have shown that following premature birth monocytes have diminished capacities for both these responses $(7,8)$.

TLRs play a crucial role in sensing bacteria and, in the case of sepsis, stimulating a pathogenic response by the innate immune system. Recognition of bacterial products (e.g., LPS) by TLRs in monocytes/macrophages leads to the production of cytokines, chemokines, and nitric oxide (9). Among them, inflammatory cytokines such as tumor necrosis factor- $\alpha$ (TNF- $\alpha$ ) play a pivotal role in the pathogenesis of early septic shock and organ dysfunction (10).

Pentoxifylline (PTX), a methylxanthin derivate and phosphodiesterase inhibitor, is a non-steroidal immunomodulating drug, which has been shown to be of potential benefit to the outcome of term and preterm neonates with sepsis and necrotizing enterocolitis. However, a current Cochrane review concluded that PTX, when used as an adjunct therapy to antibiotics in neonatal sepsis, might decrease mortality without any adverse effects (11). A recent randomized controlled trial on the use of PTX in late-onset neonatal sepsis showed no decrease in mortality, but it had several beneficial adjuvant effects in preterm infants (12). PTX is known to inhibit the synthesis of TNF- $\alpha$ (13) and other cytokines that play key roles in inflammation $(14,15)$. The inhibition of cytokine production may be mediated through the inhibition of nuclear factor (NF)-kB and c-Rel activation (16). Yet, its detailed mechanism of action remains incompletely understood, and, to our knowledge, in vitro studies on the effect of PTX on monocytes of preterm neonates have not been published so far. Given the substantial burden of sepsis and necrotizing enterocolitis in preterm neonates and the

'Division of Neonatology, Pediatric Intensive Care and Neuropediatrics, Department of Pediatrics and Adolescent Medicine, Medical University of Vienna, Vienna, Austria; ${ }^{2}$ Department of Surgery, Research Labs \& Core Facility Flow Cytometry, Medical University of Vienna, Vienna, Austria; ${ }^{3}$ Department of Pediatrics, Maastricht University Medical Center, Maastricht, The Netherlands; ${ }^{4}$ School of Oncology and Developmental Biology, Maastricht University, Maastricht, The Netherlands. Correspondence: Angelika Berger (angelika.berger@meduniwien.ac.at)

Received 24 August 2016; accepted 8 February 2017; advance online publication 24 May 2017. doi:10.1038/pr.2017.41 
potential benefit of PTX, PTX needs to be further evaluated in clinical trials and experimental studies. The purpose of this study was to assess whether PTX modulates the expression of various monocyte surface markers, cytokine production, phagocytic activity, TLR4 expression levels, and signaling in preterm and term infants compared with those in adults in an in vitro Gram-negative (LPS) sepsis model.

\section{METHODS}

\section{Study Population and Blood Collection}

Heparinized cord blood from term (37-42 weeks of gestational age) and preterm (24-32 completed weeks of gestational age) infants was obtained immediately after cesarean section (for infants' characteristics, see Table 1). Neonates with congenital malformations, signs of clinical infection, or elevated laboratory markers (elevated white blood cell count, CRP, and/or IL-8) were excluded. Additional exclusion criteria were clinical chorioamnionitis, maternal autoimmune disease, or the use of immunomodulatory drugs with the exception of prenatally administered betamethasone for the induction of fetal lung maturation during preterm labor. Peripheral blood was collected from healthy adult volunteers. The study was approved by the local ethics committee, and informed consent was obtained from pregnant women before delivery and from healthy volunteers.

\section{Reagents}

Pentoxyfylline (Trental, $100 \mathrm{mg} / 5 \mathrm{ml}$ ) was purchased from SanofiAventis Germany $\mathrm{GmbH}$ (Frankfurt, Germany) and was used at final concentrations of 20,200 , and $2,000 \mu \mathrm{g} / \mathrm{ml}$ based on previous in vitro studies (15), and blood concentrations were measured in vivo (17). LPS from Escherichia coli, Serotype R 1515 (Re) (liquid), was purchased from Alexis Biochemicals (San Diego, CA) and used at a concentration of $10 \mathrm{ng} / \mathrm{ml}$.

\section{PTX-Time and Dose-Response Studies}

For most experiments, we used whole-blood assays to mirror a most physiological environment; no further serum was added. Samples $(0.5 \mathrm{ml})$ were cultured in 24-well plates with LPS $(10 \mathrm{ng} / \mathrm{ml})$ and PTX $(20,200$, and $2,000 \mu \mathrm{g} / \mathrm{ml})$ for 4,8 , or $24 \mathrm{~h}$ at $37^{\circ} \mathrm{C}, 5 \% \mathrm{CO}_{2}$. After each incubation period, supernatants were collected, centrifuged, and stored at $-80^{\circ} \mathrm{C}$ until assayed for cytokine production.

Cord blood MNCs as wells as PBMCs were isolated from freshly drawn heparinized blood by Ficoll gradient centrifugation (FicollPaque PLUS; Amersham, GE Healthcare Life Sciences, Little Chalfont, UK) and counted on a Sysmex XS800i (Sysmex Europe $\mathrm{GmbH}$, Norderstedt, Germany). MNCs $\left(2 \times 10^{6} / \mathrm{ml}\right)$ were cultured with RPMI 1640 supplement with 10\% fetal calf serum (Invitrogen, Carlsbad, CA) without antibiotics. MNCs were stimulated with or without LPS $(10 \mathrm{ng} / \mathrm{ml})$ and with or without PTX $(200 \mu \mathrm{g} / \mathrm{ml})$ and incubated in a humidified $5 \% \mathrm{CO}_{2}$ environment at $37^{\circ} \mathrm{C}$.

\section{Monoclonal Antibodies and Flow Cytometric Analysis}

For surface staining, $50 \mu \mathrm{l}$ samples of whole blood were stained with phytoerythrin (PE) mouse anti-CD40 (clone FC3), PE-Cy5 mouse anti-CD71 (clone M-A712), fluorescein isothiocyanate (FITC) mouse anti-CD64 (clone 10.1), APC-H7 mouse anti-CD80 (clone L307.4), PE-Cy7 mouse anti-CD86 (clone 2331), Pacific Blue mouse antiCD11b/Mac-1 (clone ICRF44), and V500 mouse anti-HLA-DR (L243), all by BD Biosciences (Franklin Lakes, NJ). Anti-CD14 monoclonal antibody (mAb; My4-FITC) or Alexa Fluor 700 mouse anti-CD14 was used to define monocyte population (both from BD Biosciences). Mouse anti-CD 284 (Toll-like receptor 4) PE (clone HTA125) was purchased by eBioscience (San Diego, CA). Antibody incubation was performed on ice for $30 \mathrm{~min}$. After cell staining, erythrocytes were lysed with VersaLyse (Beckman Coulter, Brea, CA), washed, fixed with formaldehyde, and analyzed within $1 \mathrm{~h}$ by flow cytometry (LSRII, BD Biosciences, San Diego, CA). Data
Table 1. Neonatal characteristics

\begin{tabular}{lcc}
\hline & $\begin{array}{c}\text { Preterm neonates } \\
(n=14)\end{array}$ & $\begin{array}{c}\text { Term neonates } \\
(n=13)\end{array}$ \\
\hline $\begin{array}{l}\text { Gestational age } \\
\text { (weeks) }\end{array}$ & $28.8 \pm 3.14$ & $38.78 \pm 1.13$ \\
Infant body mass (g) & $1,179 \pm 427$ & $3,340 \pm 422$ \\
Male/female & $8 / 14$ & $6 / 13$ \\
Multiples & $4 / 14$ & $2 / 13$ \\
5-Min Apgar score & $8.38 \pm 0.65$ & $9.92 \pm 0.28$ \\
\hline
\end{tabular}

Data are presented as numbers or as mean values \pm SD.

analysis was performed with the FlowJo software (TreeStar, Ashland, OR).

\section{Intracellular Cytokine Detection}

For intracellular TNF- $\alpha$ detection, PBMCs were incubated for $5 \mathrm{~h}$ with LPS $(10 \mathrm{ng} / \mathrm{ml})$ or LPS and PTX $(200 \mu \mathrm{g} / \mathrm{ml})$ in the presence of Brefeldin A (eBioscience), washed with cold phosphate-buffered saline without $\mathrm{Ca}^{2+} / \mathrm{Mg}^{2+}$, and subsequently stained using IntraPrep (Beckman Coulter), according to the manufacturer's instructions. Monocytes were characterized by CD14 FITC (incubation for $15 \mathrm{~min}$ at room temperature), and intracellular TNF- $\alpha$ was detected with an anti-TNF- $\alpha$ PE mAb (clone MAb11; eBioscience). A corresponding isotype control was used in each experiment. TNF- $\alpha$ levels were calculated as fold change of MFI values by the formula (MFI stimulated $_{\text {I }}$ $\mathrm{MFI}_{\text {unstimulated }}$.

\section{Phospho-Staining}

Samples of $200 \mu \mathrm{l}$ human whole blood were either treated with vehicle (RPMI 1640) or PTX $(200 \mu \mathrm{g} / \mathrm{ml})$ for $24 \mathrm{~h}$. Thereafter, blood was stimulated with LPS $(10 \mathrm{ng} / \mathrm{ml})$ or left untreated at $37^{\circ} \mathrm{C}$ in sterile pyrogen-free tubes. After stimulation, red cell blood cell lysis and fixation of phospho-epitopes were performed simultaneously using BD Phosflow Lysis/Fix Buffer for $10 \mathrm{~min}$ at $37^{\circ} \mathrm{C}$ (BD Biosciences), followed by permeabilization with ice-cold Perm Buffer II (BD Biosciences, Franklin Lakes, NJ) for $30 \mathrm{~min}$. Staining with anti-phospho-ERK1/2 (T202/Y204) PE and anti-phospho-NF- $\mathrm{BB}$ p65 (pS529) (both from BD Biosciences) was performed according to the BD Phosflow protocol (BD Biosciences). Briefly, cells were washed twice with washing buffer and phospho-specific mAbs were added. Anti-CD14 FITC was added simultaneously. The mixture was incubated for $30 \mathrm{~min}$ at room temperature. The MFI was used to determine the fold change on TLR-ligand stimulation ( $\left.\mathrm{MFI}_{\text {stimulated }} / \mathrm{MFI}_{\text {unstimulated }}\right)$.

\section{Cytokine Quantification in Supernatants after Whole-Blood Stimulation}

A volume of $0.5 \mathrm{ml}$ of whole blood was stimulated with LPS $(10 \mathrm{ng} / \mathrm{ml})$ or LPS+PTX $(20,200$, or $2,000 \mu \mathrm{g} / \mathrm{ml})$ as indicated. After 4,8 , and $24 \mathrm{~h}$ of stimulation, samples were centrifuged. Cell-free supernatants were collected and frozen at $-80^{\circ} \mathrm{C}$ until further analyzed. The level of cytokines in the culture supernatants were determined using a Bead Array System (Human Inflammatory Cytokines Kit, BD Biosciences, Franklin Lakes, NJ) according to the manufacturer's protocol (BD Pharmingen, Franklin Lakes, NJ). Samples were assayed using flow cytometry (LSRII, BD Biosciences). Results were analyzed using BD CBA software version 3.0. (BD Biosciences). The limits of sensitivity were $3.3 \mathrm{pg} / \mathrm{ml}$ for IL-10, $2.5 \mathrm{pg} / \mathrm{ml}$ for IL-6, $7.2 \mathrm{pg} / \mathrm{ml}$ for IL1- $\beta, 1.9 \mathrm{pg} / \mathrm{ml}$ for IL- $12 \mathrm{p} 40$, and $3.7 \mathrm{pg} / \mathrm{ml}$ for TNF- $\alpha$.

\section{Phagocytosis}

Phagocytosis was determined by incubating $100 \mu \mathrm{l}$ of whole blood for $30 \mathrm{~min}$ with LPS $(10 \mathrm{ng} / \mathrm{ml})+$ PTX $(20,200$, or $2,000 \mu \mathrm{g} / \mathrm{ml})$ at $37^{\circ} \mathrm{C}$ with non-opsonized, FITC-conjugated E. coli, according to the 
a

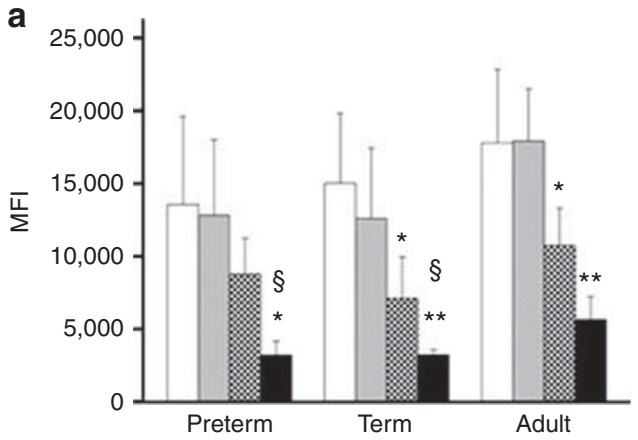

c

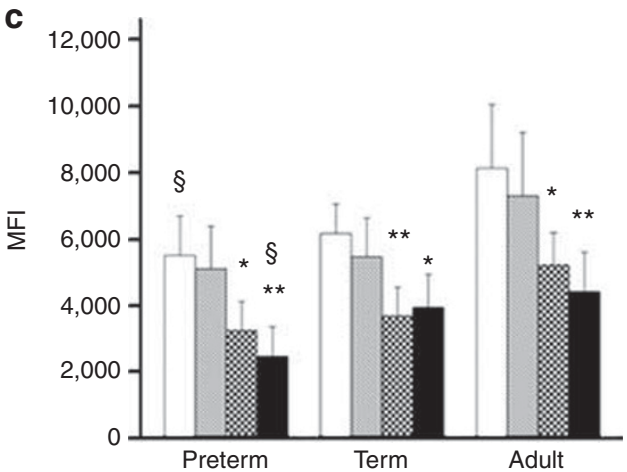

e

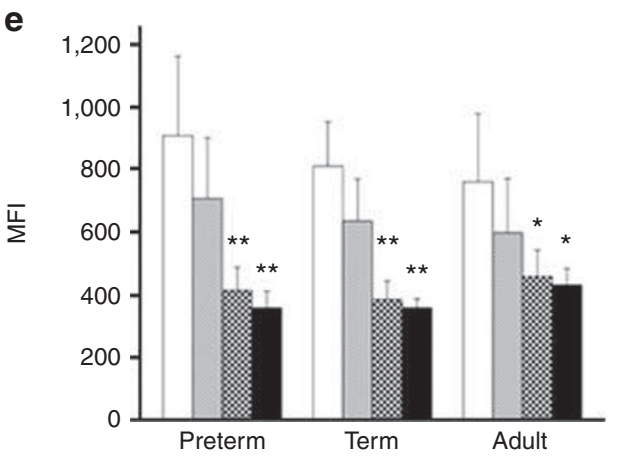

g

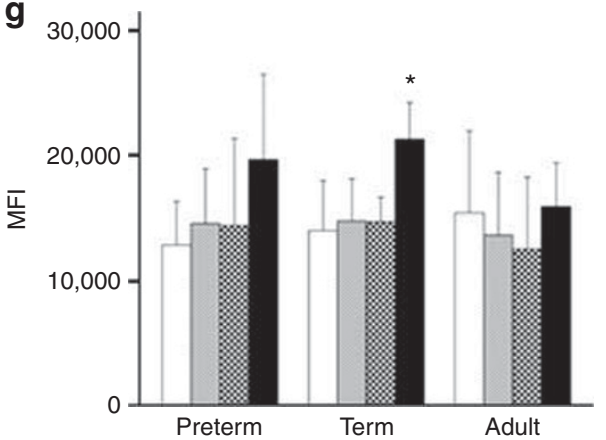

b

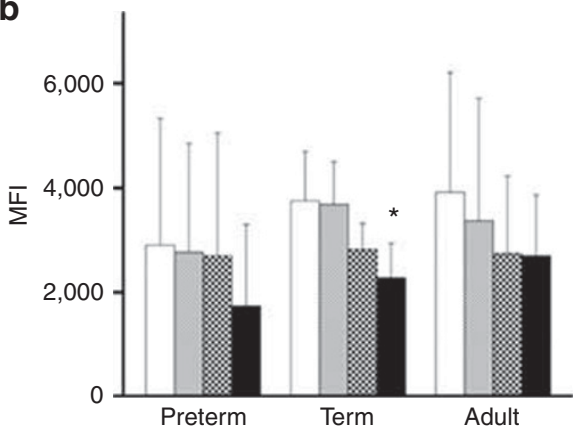

d

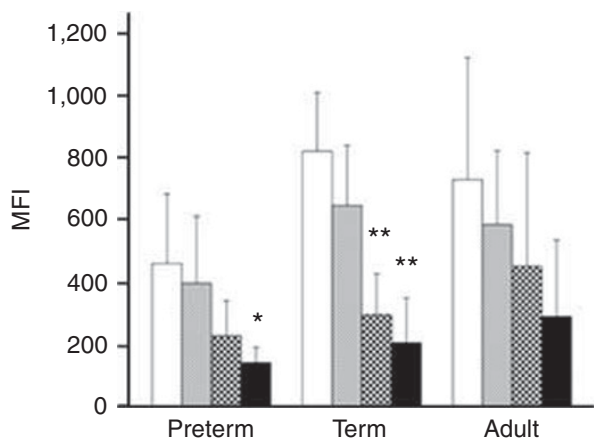

f

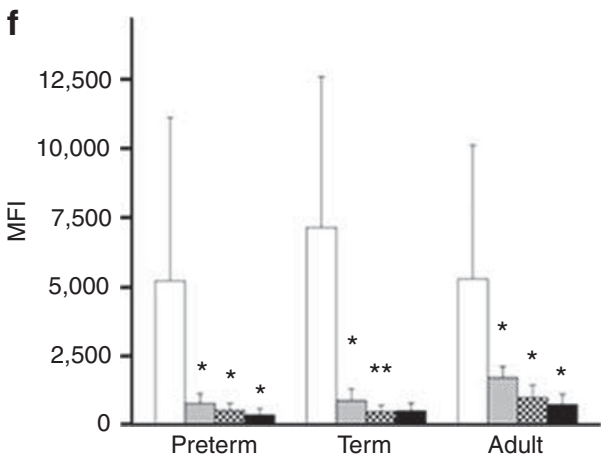

h

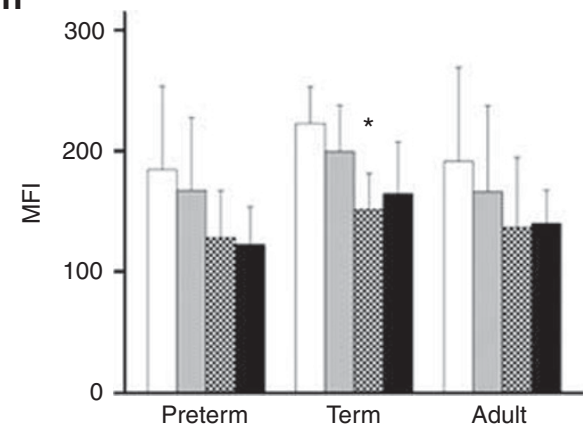

Figure 1. Pentoxifylline (PTX) differentially alters surface expression of activation and maturation markers in monocytes of term and preterm neonates compared with that in adults. To determine the effect of PTX on monocyte phenotypic maturation, whole blood was incubated with LPS $(10 \mathrm{ng} / \mathrm{ml})$ for $24 \mathrm{~h}$ with or without addition of different concentrations of PTX. White-filled columns, LPS only; black- and white-striped columns, LPS +PTX $20 \mu \mathrm{g} / \mathrm{ml}$; black- and white-chequered columns, LPS+PTX $200 \mu \mathrm{g} / \mathrm{ml}$; and black-filled columns LPS+PTX $2000 \mu \mathrm{g} / \mathrm{ml}$. Cell surface molecules CD14, HLA-DR, CD11b, CD71, CD64, CD80, CD86, and CD40 were examined on CD14-positive monocytes by flow cytometry. Surface molecule expression is presented as mean fluorescence intensities (MFIs). Mean data ( \pm SD) from adults, term, and preterm neonates ( $n=5$ for each group) are shown. A statistical analysis was performed by analysis for variance. Tukey honest significant difference post hoc test analysis showed a significant dose-dependent downregulation for (a) CD14, (b) HLA-DR, (c) CD11b, (d) CD71, (e) CD64, (f) CD80, and (h) CD40 ( $\left.{ }^{*} P<0.05,{ }^{* *} P<0.005\right)$; only for (g) CD86, an upregulation was found $(* P<0.05)$. Compared with adults, preterm neonates had significantly lower CD14 and CD11b expression levels when PTX $(2,000 \mu \mathrm{g} / \mathrm{ml})$ was added $\left({ }^{\S} P<0.05\right)$. 

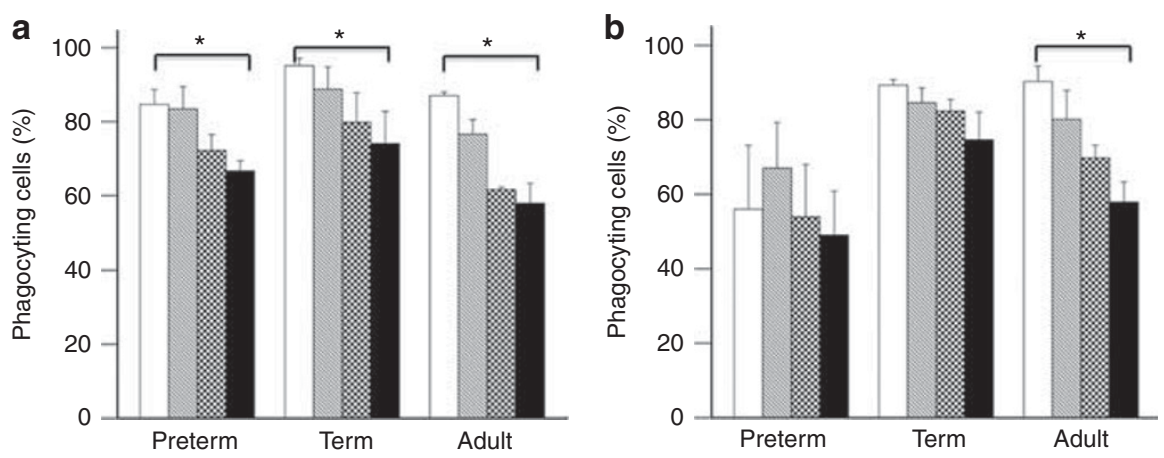

Figure 2. E. coli phagocytosis of monocytes and granulocytes from cord blood of term and preterm neonates and peripheral blood of adults were investigated by flow cytometry. The effect of pentoxifylline (PTX; mean values \pm 1 SEM) on fluorescein isothiocyanate-labeled non-opsonized $E$. coli phagocytosis in preterm and term neonates compared with that in adults was determined. White-filled columns, LPS only; black- and white-striped columns, LPS+PTX $20 \mu \mathrm{g} / \mathrm{ml}$; black- and white-chequered columns, LPS+PTX $200 \mu \mathrm{g} / \mathrm{ml}$; and black-filled columns LPS+PTX $2000 \mu \mathrm{g} / \mathrm{ml}$. Results are given as percentage of phagocyting cells. Monocytes were characterized by CD14 and granulocytes were identified by side scatter. The analysis for phagocytosis in monocytes (a) showed a dose-dependent decrease of phagocytosis under PTX treatment without age-dependent differences. In granulocytes $(\mathbf{b})$, the suppressive effect was less pronounced in neonates, but reached significance in adults $\left({ }^{*} P<0.05\right)$.

manufacturer's protocol of Phagotest (Glycotope Biotechnology, Heidelberg, Germany). Monocytes were stained with APC anti-CD14 $\mathrm{mAb}$ (clone M5E2 by BD), and red cells were lysed with VersaLyse (Beckman Coulter). Cells were washed three times in Hank's Balanced Salt Solution. The MFI of ingested E. coli bacteria from $5 \times 10^{3} \mathrm{CD} 4^{+}$monocytes and granulocytes that had been gated by side scatter was determined by flow cytometry, and MFI of ingested FITC-labeled E. coli was analyzed.

\section{Cell Viability Assay}

A PE Annexin V Apoptosis Detection Kit I by BD Pharmingen assessed cell viability according to the manufacturer's protocols. In brief, $300 \mu \mathrm{l}$ of whole blood (peripheral blood in adults and cord blood in neonates) was incubated with PTX $(2,000 \mu \mathrm{g} / \mathrm{ml})$ for $24 \mathrm{~h}$. Thereafter, cells were washed with phosphate-buffered saline, and they were resuspended in binding buffer. Staining was performed with CD14 FITC, 7-amino-actinomycin, and PE-labeled Annexin V for $20 \mathrm{~min}$ at room temperature. Red cells were lysed with VersaLyse. Cells were washed again with phosphate-buffered saline and fixed with $4 \%$ formaldehyde, and fluorescence-activated cell sorting analysis was conducted within $1 \mathrm{~h}$.

Isolation of Monocytes, RNA Extraction, and cDNA Synthesis For TLR4-mRNA quantification, monocytes were separated by density gradient centrifugation and by means of magnetic cell sorting (Monocyte isolation kit II; Milteny Biotec, Bergisch Gladbach, Germany). Monocyte purity of negatively selected cells was usually $\sim 95 \%$, as determined by flow cytometry. Cells $\left(1 \times 10^{6} / \mathrm{ml}\right)$ were seeded in culture plates and cultured in RPMI 1640 supplemented with $10 \%$ fetal calf serum in the presence or absence of LPS (10 ng/ $\mathrm{ml})$ or LPS+PTX $(200 \mu \mathrm{g} / \mathrm{ml})$, for $6 \mathrm{~h}$. Thereafter, total RNA was prepared using the RNeasy mini kit (Qiagen, Germantown, MD) according to the manufacturer's instruction. RNA purity and quantity were determined using Nanodrop (Thermo Fisher, Waltham, MA).

The ABI PRISM 7500HT sequence detection system (Applied Biosystems, Life Technologies, Grand Island, NY) was used for quantitative reverse-transcriptase PCR analysis. Primer-probe sets for TLR4 FAM and 18S rRNA VIC were obtained, pre-designed from Applied Biosystems, and tested for primer efficacy (Gene Expression Assays Hs99999901_s1 18S VIC, Hs00152939_m1 TLR4 FAM). Multiplex amplification was carried out in a total volume of $20 \mu \mathrm{l}$ for 45 cycles of $3 \mathrm{~s}$ at $95^{\circ} \mathrm{C}$ and $30 \mathrm{~s}$ at $60^{\circ} \mathrm{C}$. Initial denaturation was performed for $3 \mathrm{~min}$ at $95^{\circ} \mathrm{C}$. Target gene expression was normalized to $18 \mathrm{~s}$ rRNA housekeeping gene expression. Normalized target gene expression was analyzed by the $\Delta \Delta$ comparative threshold method and calculated as $x$ fold expression TLR4.

\section{Statistical Methods}

Infants were enrolled into the study over a 12-month period. Statistical analysis was performed with SPSS software (SPSS 15.0 for Windows, Armonk, NY). Data were analyzed using one-way analysis for variance in accordance to Tukey $B$ or the two-tailed $t$-test with Bonferroni correction. A probability of $P<0.05$ was considered statistically significant.

\section{RESULTS}

Effects of LPS and PTX on the Expression of Activation and Maturation Markers in Monocytes of Preterm and Term Neonates Compared with Adults

Monocytes were identified by CD14. LPS led to an upregulation of most surface markers after $24 \mathrm{~h}$ compared with medium-only-treated monocytes. For reasons of clarity, the LPS-only group was set as a control in Figure $\mathbf{1 .}$

CD14 was downregulated in a dose-dependent manner by PTX in all age groups (Figure 1a). In the highest PTX concentration tested $(2,000 \mu \mathrm{g} / \mathrm{ml})$, monocytes of term and preterm infants ended up with a significantly lower CD14 expression compared with that in monocytes of adults $(P<0.05)$.

HLA-DR expression was only moderately influenced by PTX. Compared with controls, a decrease in HLA-DR level was observed in all age groups, which only reached statistical significance in the group of term infants at the highest PTX concentration $(P<0.05)$. Similar results were found for CD40 (Figure 1h) and CD54 expressions, but with the latter decrease not reaching statistical significance (results not shown).

CD11b, a polypeptide $\alpha$-chain linked to the $\beta 2$-subunit of CD18, was significantly downregulated by PTX. This effect was most pronounced in preterm neonates reaching a statistically significant difference compared with adult CD11b expression $(P<0.05$, Figure 1c). 
CD71, also known as the transferrin receptor, is a marker for the activation of monocytes. CD71 was statistically significantly downregulated on monocytes from term and preterm neonates and to a lesser extent in adults (Figure 1d).

The expression of CD64 on monocytes was downregulated in a dose-dependent manner in all age groups without any significant age-dependent differences (Figure 1e).

The co-stimulatory molecules, CD80 and CD86, showed a divergent pattern upon PTX stimulation. CD80 showed a clear dose-dependent downregulation (Figure 1f) in all age groups, whereas CD86 was upregulated in the highest dose of PTX tested, which reached significance only in term neonates because of high SD in preterm infants. Adult levels of CD86 expression were not significantly influenced (Figure 1g).

\section{Analysis of the Effects of PTX on Phagocytosis Activity}

PTX reduced phagocytosis activity in monocytes in a dosedependent manner, which was most pronounced in adults (Figure 2a). Similarly, we found a statistically significant reduction of phagocytic activity of PTX-incubated granulocytes in adults, but there was only a tendency for it in term and preterm neonates. However, granulocytes of preterm neonates had lower phagocytic activity at baseline and showed only a moderate decline in phagocytic activity after PTX incubation (Figure 2b).

\section{Effect of PTX on TNF-a Production}

For intracellular TNF- $\alpha$ quantification, peripheral blood mononuclear cells (PBMCs) were left untreated or stimulated with LPS $(10 \mathrm{ng} / \mathrm{ml}) \pm$ PTX $(200 \mu \mathrm{g} / \mathrm{ml})$ in the presence of an inhibitor of intracellular protein transport (Brefeldin A) for $5 \mathrm{~h}$. Thereafter, intracellular TNF- $\alpha$ was measured in CD14positive monocytes by flow cytometry (Figure 3a). PTX reduced intracellular TNF- $\alpha$ production (measured by median fluorescence intensity (MFI) fold change) in a consistent way as shown in Figure 3c (mean reduction of MFI index for adult: $39.0 \pm 9.3 \%$, term: $44.4 \pm 19.3 \%$, preterm: $37.4 \pm 16.3 \%$, $P<0.05$ for all age groups). The reduction of TNF- $\alpha-$ producing cells was statistically significant in all age groups (adult: $26.8 \pm 16.4 \%$, term: $43.8 \pm 21.4 \%$, preterm: $32.2 \pm 14.9 \% ; \quad(P<0.05$, Figure $3 \mathbf{b})$. No age-dependent difference in the ability of PTX to reduce TNF was found.

In a different experiment, TNF- $\alpha$ was measured in the supernatant of a whole-blood culture stimulated with LPS $(10 \mathrm{ng} / \mathrm{ml})$ in the presence and absence of PTX $(200 \mu \mathrm{g} / \mathrm{ml})$ for 4,8 , and $24 \mathrm{~h}$. TNF- $\alpha$ levels of controls (unstimulated) were low or not detectable at all (Table 2). As shown before, upon LPS stimulation, monocytes of adults produced significantly more TNF- $\alpha$ than those of neonates at all time intervals studied. PTX led to a highly significant reduction of TNF- $\alpha$ levels in the supernatant in all age groups in absolute (Table 2) and relative numbers (Figure 3d), but again no agedependent difference in the ability of PTX to reduce TNF- $\alpha$ was found.
Effect of PTX on the Production of IL-10, IL-1- $\beta$, IL-6, and IL-8 Activation by LPS clearly induced an increase in all cytokines investigated (Table 2) except in IL-12 production, for which reason we excluded IL-12 from further investigations. As demonstrated in Figure 4a, PTX showed an age-dependent difference for IL-10 production. We found a highly significant reduction of IL-10 in term $(28.2 \pm 16.1 \%)$ and preterm infants $(11.2 \pm 8.4 \%)$ after $4 \mathrm{~h}$ of incubation, whereas adult IL-10 levels were not influenced $(119.0 \pm 28.2 \%)$ compared with those in LPS-stimulated controls. IL-10 levels stayed significantly reduced after $8 \mathrm{~h}$ in term $(9.2 \pm 4.8 \%)$ and preterm $(13.0 \pm 8.8 \%)$ samples, whereas adult levels were moderately decreased $(48.7 \pm 19.6 \%)$. These age-dependent differences disappeared after $24 \mathrm{~h}$.

LPS-induced secretion of IL-1- $\beta$ and IL-6 levels was significantly reduced under the influence of PTX after 4 and $8 \mathrm{~h}$ in adults and neonates without any differences between the groups (Figure $\mathbf{4 b}, \mathbf{c}$ ). This suppressive effect of PTX disappeared after $24 \mathrm{~h}$. A significant IL-8 suppression was only observed in preterm neonates after $4 \mathrm{~h}$ and in term neonates after $8 \mathrm{~h}$ (Figure $4 \mathrm{~d}$ ). We found no significant difference in IL-1- $\beta$, IL-6, and IL-8 reduction levels between the age groups investigated, although there was a trend toward a stronger suppression of IL-1- $\beta$ in preterm infants compared with that in adults $(P=0.058)$ after $8 \mathrm{~h}$ (Figure $4 \mathrm{~b})$.

\section{Effects of LPS and PTX on TLR4 Surface Expression and mRNA Level}

TLR4 expression was analyzed on monocytes characterized by CD14 after incubation of whole blood for $24 \mathrm{~h}$ with LPS $(10 \mathrm{ng} / \mathrm{ml})$ and PTX as indicated and measured by flow cytometry (Figure 5a). TLR4 expression was reduced in a dose-dependent manner in adults, reaching statistical significance in monocytes treated with PTX $(200 \mu \mathrm{g} / \mathrm{ml})$ and PTX $(2,000 \mu \mathrm{g} / \mathrm{ml})$. This effect was less pronounced in preterm neonates (Figure $5 \mathrm{~b}$ ).

We also tested whether TLR4 expression was downregulated in a PBMC culture. As shown in Figure 5c, TLR4 downregulation started after $12 \mathrm{~h}$, although the effect was less pronounced in the PBMC culture than in whole blood and did not reach statistical significance.

Finally, we investigated TLR4 on the transcriptional level. As shown in Figure 5d, upregulation of messenger RNA (mRNA) level of TLR4 by LPS stimulation was significantly reduced if PTX was co-administrated.

\section{Analysis of NF-kB Phosphorylation}

This experiment was only performed in adults and preterm neonates, as we did not expect differences between term and preterm neonates based on previous results (3). Stimulation of whole blood with PTX for $24 \mathrm{~h}$ resulted in a significantly reduced upregulation of phospho-NF- $\kappa \mathrm{B}$ (Figure 6a,c) and phospho-ERK1/2 (Figure $6 \mathbf{b}, \mathbf{d}$ ) upon LPS stimulation $(10 \mathrm{ng} / \mathrm{ml})$ in monocytes of preterm neonates and adults. We observed no age-dependent differences in this PTX effect. In monocytes that had not been stimulated with LPS, PTX did 

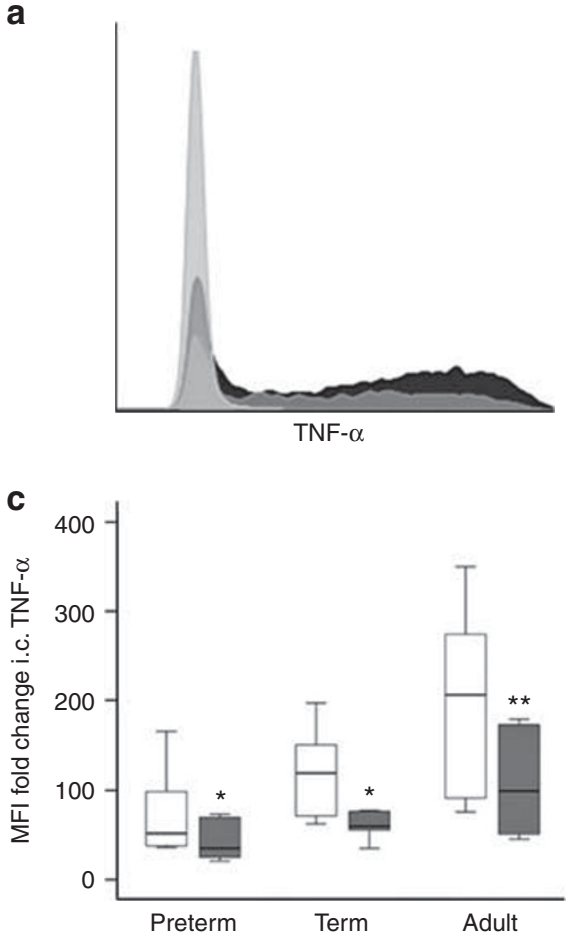

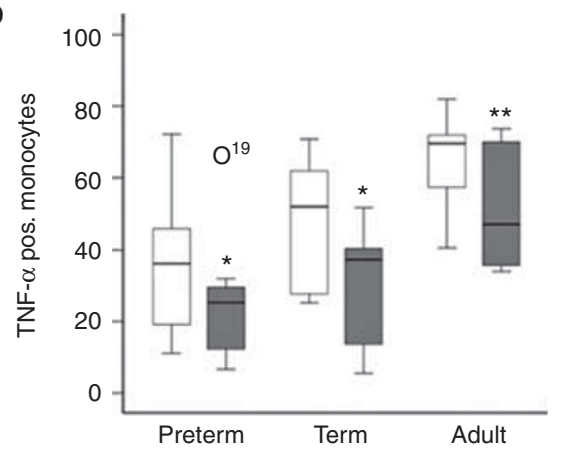

d

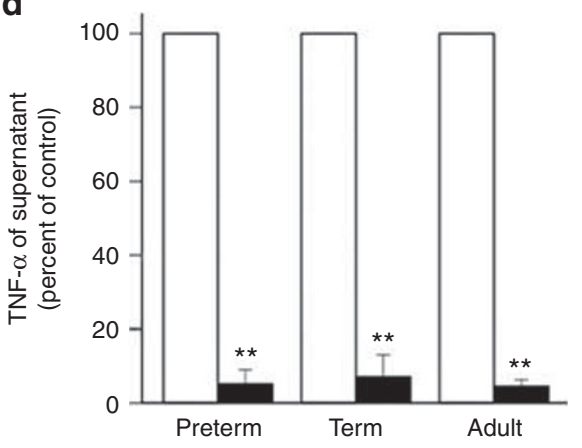

Figure 3. Effect of pentoxifylline (PTX) on tumor necrosis factor-a (TNF-a) production in monocytes of term and preterm neonates compared with that in adults. For intracellular TNF-a cytokine staining, peripheral blood mononuclear cells were incubated in the presence of LPS \pm PTX (200 $\mu \mathrm{g} / \mathrm{ml}$ ) for $5 \mathrm{~h}$, collected thereafter, and stained for CD14 and TNF- $a$. Upon LPS challenge, TNF-a was found exclusively in monocytes. Stimulation with LPS induced a robust TNF-a production in all age groups. (a) Respective histogram. Light gray indicates medium only, medium gray indicates LPS+PTX, dark gray indicates isotype control, and black indicates LPS only. PTX led to a significant decrease of TNF-a fold change compared with LPS-

stimulated control (c) without differences seen in the potency of this PTX effect. The percentage of intracellular TNF-a positive monocytes was also affected by PTX in a significant way without any age-specific differences (b). Blank boxes indicate treatment with LPS only, and filled boxes indicate LPS+PTX. The experiment with monocytes was derived from seven adults, five term infants, and seven preterm infants $\left({ }^{*} P<0.05\right.$, $\left.{ }^{* *} P<0.005\right)$. For the measurement of TNF-a in the supernatant, cord blood (neonates) and peripheral blood (adults) were cultured with LPS (10ng/ml) in the absence or presence of PTX $(200 \mu \mathrm{g} / \mathrm{ml})$. After incubation, cell-free supernatants were collected and analyzed for TNF-a levels by a capture bead array. Blank boxes indicate treatment with LPS only, and filled boxes indicate LPS+PTX. TNF-a production was significantly suppressed in all age groups $\left({ }^{* *} P<0.005\right)$ after (d) $8 \mathrm{~h}$.

not significantly influence expression levels of phospho-NF$\kappa \mathrm{B}$ and phopho-ERK1/2.

\section{No Apoptotic Effect of PTX}

We found no apoptotic effect of PTX on monocytes treated with the highest PTX dose tested $(2,000 \mu \mathrm{g} / \mathrm{ml})$ for up to $24 \mathrm{~h}$ using Annexin- $\mathrm{V}$ as the detection method (data not shown).

\section{DISCUSSION}

Preterm neonates are highly susceptible to bacterial infections, ultimately leading to sepsis. In sepsis, the initial proinflammatory response can be overwhelming, suggesting that the innate immune response determines, next to the pathogen itself, the outcome of a septic neonate. The mechanistic effects of immunomodulatory agents in neonates and preterm infants are yet to be established.

This is the first study to investigate the immunomodulatory effects of PTX on monocytes from preterm neonates in vitro. The results demonstrate that PTX exerts a dose-dependent downregulation of many surface markers and TLR4 expression. Our results support the anti-inflammatory effects of PTX found in vivo in septic preterm neonates studied by Lauterbach et al. (18) and described for adult PBMCs in vitro (19). Using a whole-blood model with final concentrations of PTX also measured in preterm neonates in vivo (17), we tried to resemble a most physiological condition.

The concentration of PTX measured in vivo in preterm neonates after infusion of PTX $(5 \mathrm{mg} / \mathrm{kg} / \mathrm{h})$ varied from 213 to $23,859 \mathrm{ng} / \mathrm{ml}(($ ref. 17)). This suggests that PTX clearance may be age-related, although other variables may have an effect including the method of administration. Regarding dosage, no adverse effects of PTX were observed in clinical trials in premature infants $(17,18)$. This is consistent with the in vitro results of our study, demonstrating no toxic effect of PTX on monocytes treated with up to $2,000 \mu \mathrm{g} / \mathrm{ml}$.

PTX significantly diminished plasma TNF- $\alpha$ and IL-6 levels. We found no age-dependent differences in the ability of PTX to reduce TNF- $\alpha$ production. In contrast to Lauterbach, but in line with the adult in vitro data (14), our results demonstrate a suppressive effect for IL-1 $\beta$ and IL-8 (Figure 4b,d). 
Table 2. Production of cytokines by cord blood (preterm and term infants) and adult blood samples after incubation for 4,8 , and $24 \mathrm{~h}$

\begin{tabular}{|c|c|c|c|c|c|c|c|c|c|c|c|}
\hline & & \multicolumn{3}{|c|}{ Unstimulated } & \multicolumn{3}{|c|}{ LPS stimulated } & \multicolumn{3}{|c|}{ LPS+PTX stimulated } & \\
\hline & & Preterm & Term & Adult & Preterm & Term & Adult & Preterm & Term & Adult & \\
\hline Incubation period & Cytokine & & & & & & & & & & \\
\hline \multicolumn{12}{|l|}{$4 \mathrm{~h}$} \\
\hline & TNF (pg/ml) & $5 \pm 4$ & ND & ND & $206 \pm 183$ & $216 \pm 266$ & $4,802 \pm 6776$ & $7 \pm 7$ & $6 \pm 5$ & $200 \pm 289$ & \\
\hline & IL-10 (pg/ml) & ND & ND & ND & $60 \pm 13$ & $25 \pm 19$ & $14 \pm 14$ & $7 \pm 6$ & $9 \pm 9$ & $15 \pm 12$ & \\
\hline & IL-6 (pg/ml) & $1,345 \pm 1,542$ & $36 \pm 56$ & ND & $17,986 \pm 10,117$ & $39,496 \pm 25,865$ & $37,196 \pm 25,600$ & $3,901 \pm 1,344$ & $17,968 \pm 23,522$ & $16,955 \pm 14,459$ & \\
\hline & $\mathrm{IL}-1 \beta(\mathrm{pg} / \mathrm{ml})$ & $12 \pm 13$ & ND & ND & $350 \pm 186$ & $368 \pm 176$ & $570 \pm 394$ & $43 \pm 26$ & $81 \pm 82$ & $181 \pm 66$ & \\
\hline & IL-8 (pg/ml) & $1,856 \pm 847$ & $1,242 \pm 1967$ & $73 \pm 41$ & $20,898 \pm 11,237$ & $28,448 \pm 5646$ & $9,357 \pm 2016$ & $7,573 \pm 3,763$ & $16,803 \pm 17,300$ & $7,432 \pm 2,307$ & \\
\hline \multicolumn{12}{|l|}{$8 \mathrm{~h}$} \\
\hline & TNF (pg/ml) & $7 \pm 5$ & ND & ND & $277 \pm 303$ & $354 \pm 275$ & $3,812 \pm 2,190$ & $27 \pm 44$ & ND & $191 \pm 165$ & \\
\hline & IL-10 (pg/ml) & ND & ND & ND & $141 \pm 92$ & $321 \pm 267$ & $149 \pm 92$ & $141 \pm 92$ & $23 \pm 8$ & $66 \pm 41$ & \\
\hline & IL-6 (pg/ml) & $1,011 \pm 1,694$ & $36 \pm 56$ & $7 \pm 1$ & $28,195 \pm 13,275$ & $70,289 \pm 18,754$ & $52,129 \pm 16,629$ & $28,195 \pm 13,275$ & $27,117 \pm 14,158$ & $26,883 \pm 18,119$ & \\
\hline & $\mathrm{IL}-1 \beta(\mathrm{pg} / \mathrm{mL})$ & $11 \pm 18$ & ND & ND & $403 \pm 285$ & $1,525 \pm 492$ & $3,188 \pm 1,543$ & $403 \pm 285$ & $208 \pm 82$ & $790 \pm 450$ & \\
\hline & IL-8 (pg/ml) & $5,579 \pm 7,121$ & $19,03 \pm 3,008$ & $89 \pm 53$ & $31,407 \pm 19,884$ & $78,057 \pm 28,250$ & $9,379 \pm 3,750$ & $11,966 \pm 9,352$ & $32,650 \pm 17,216$ & $9,299 \pm 4,273$ & \\
\hline \multicolumn{11}{|l|}{$24 \mathrm{~h}$} & \\
\hline & TNF (pg/ml) & $5 \pm 7$ & ND & ND & $86 \pm 144$ & $4 \pm 2$ & $1,969 \pm 1,550$ & $21 \pm 34$ & ND & $6 \pm 8$ & e \\
\hline & IL-10 (pg/ml) & ND & ND & ND & $337 \pm 171$ & $1,362 \pm 1,719$ & $988 \pm 270$ & $337 \pm 134$ & $36 \pm 21$ & $132 \pm 75$ & $\bar{y}$ \\
\hline & IL-6 (pg/ml) & $863 \pm 1,491$ & $78 \pm 100$ & ND & $20,344 \pm 8,873$ & $65,626 \pm 22,951$ & $57,310 \pm 10,469$ & $20,344 \pm 8,874$ & $42,099 \pm 28,715$ & $43,095 \pm 22,148$ & 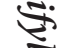 \\
\hline & $\mathrm{IL}-1 \beta(\mathrm{pg} / \mathrm{ml})$ & $8 \pm 13$ & ND & ND & $393 \pm 329$ & $1,958 \pm 269$ & $3,865 \pm 1,617$ & $393 \pm 329$ & $224 \pm 112$ & $1,095 \pm 647$ & \\
\hline & IL-8 (pg/ml) & $1,623 \pm 1,799$ & $828 \pm 821$ & $618 \pm 825$ & $55,059 \pm 35,192$ & $84,050 \pm 20,620$ & $25,637 \pm 4043$ & $52,987 \pm 46502$ & $37,257 \pm 18,308$ & $37,402 \pm 10,113$ & \\
\hline \multicolumn{12}{|c|}{$\begin{array}{l}\text { IL, interleukin; ND, not determined; PTX, pentoxifylline; TNF, tumor necrosis factor. } \\
\text { Data are represented as mean values } \pm \text { SD. }\end{array}$} \\
\hline
\end{tabular}



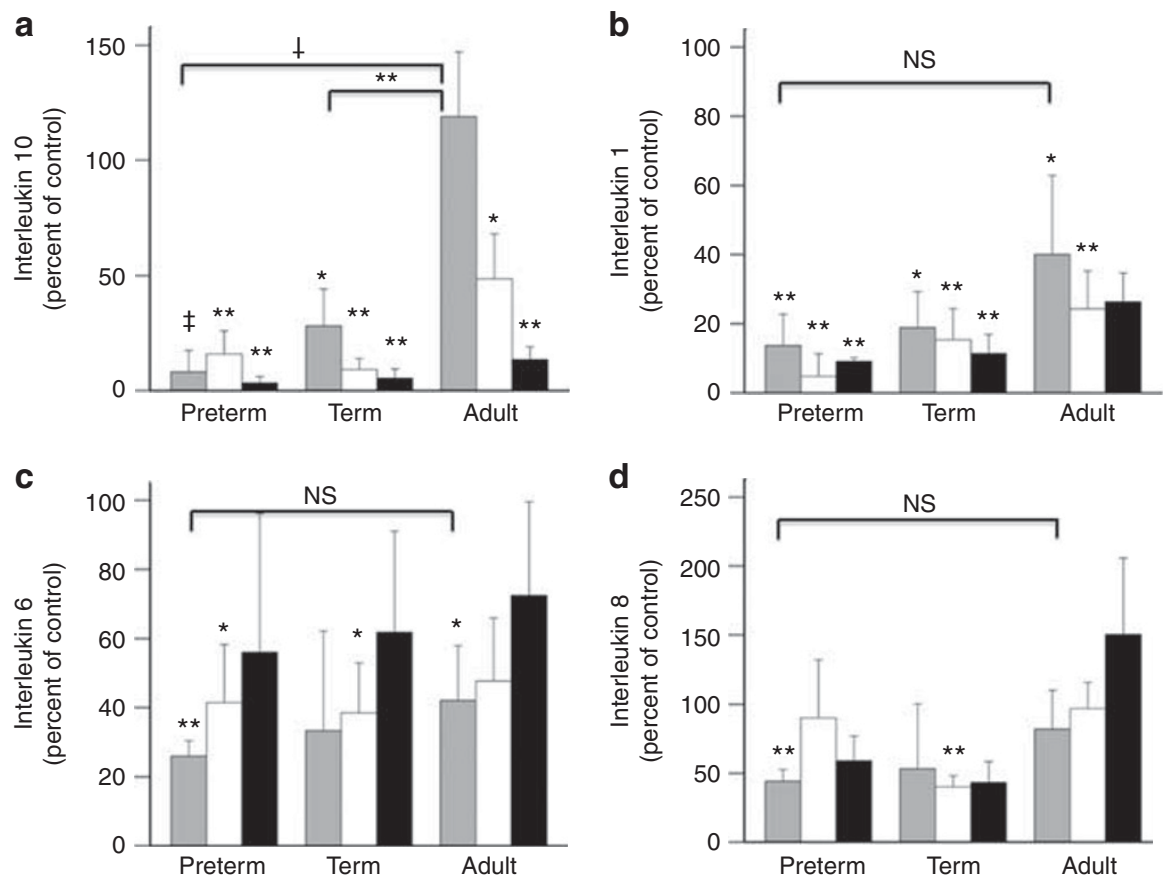

Figure 4. Effect of pentoxifylline (PTX) on interleukin (IL)-1, IL-6, IL-8, and IL-10 production in whole blood of term and preterm neonates compared with that in adults. Cord blood (neonates) and peripheral blood (adults) were cultured with LPS (10 ng/ml) in the absence or presence of PTX $(200 \mu \mathrm{g} / \mathrm{ml})$. After incubation for 4 (blank), 8 (gray), or 24 (black) hours, cell-free supernatants were collected and analyzed for IL-10 (a), IL-1 $\beta$ (b), IL-6 (c), and IL-8 (d) levels by a capture bead array. PTX led to a significant reduction of IL-10 levels after $4 \mathrm{~h}$ in preterm neonates $\left({ }^{\ddagger} P<0.0001\right)$ and to a lesser extent in term neonates $\left({ }^{*} P>0.05\right)$. In contrast, IL-10 production in adults was not influenced after $4 \mathrm{~h}$. This age-dependent difference was highly significant for preterm $\left({ }^{\ddagger} P<0.0001\right)$ and term neonates $(P<0.005)$. After $8 \mathrm{~h}$, the IL-10 production in adults decreased statistically $(* P<0.05)$, whereas in preterm and term neonates, the suppressive effect of PTX was still more pronounced $(* * P<0.005)$. After $24 \mathrm{~h}$, IL-10 production was significantly reduced in all groups without any age differences. PTX also reduced IL-1, IL-6, and IL-8 levels significantly in neonates and adults (except for IL-8). There was a tendency for a more pronounced effect in preterm neonates compared with that in adults $(P=0.08$ after $4 \mathrm{~h} ; P=0.058$ after $8 \mathrm{~h}$ ). Data from adults, term, and preterm neonates are shown. Bars show mean values \pm SD of the cytokine levels (\%) compared with LPS-stimulated samples. ${ }^{*} P<0.05,{ }^{* *} P<0.005,{ }^{\ddagger} P<0.0001$.

Notably, our results indicate an age-dependent, divergent influence of PTX on IL-10 production (Figure 4a). Adult IL-10 levels were not influenced by PTX after 4 and $8 \mathrm{~h}$ but showed a significant decrease after $24 \mathrm{~h}$, as has been published earlier (14). In neonates, IL-10 levels were already significantly reduced by $4 \mathrm{~h}$ and stayed reduced at 8 and $24 \mathrm{~h}$. These results suggest that PTX may not only inhibit the Th1 cytokine profile but also suppress IL-10, an anti-inflammatory cytokine in term and preterm neonates at an early stage. Previous in vitro studies of murine macrophages confirm our findings of the ability of PTX to reduce IL-10 production (20), whereas other in vivo studies showed an increase of IL-10 mRNA in human blood mononuclear cells (MNCs) (21) after PTX treatment. The role of IL-10 has been increasingly recognized in modulating the immunologic response to sepsis. IL-10 limits inflammation by inhibiting APC and effector T-cell function (22). In an experimental model, it was shown that the administration of recombinant murine IL-10 protects from lethal endotoxemia, even when IL-10 was injected $30 \mathrm{~min}$ after LPS administration (23). In contrast, the immunoneutralization of IL-10 led to increased levels of TNF and IL-6 in mice (24). Despite these clear protective effects, IL-10 actions might not always be beneficial, as studies found that IL-10 levels did correlate with mortality in pediatric intensive care unit patients (24).

A reduced phagocytic activity in sepsis has also been shown to be associated with a poor outcome in adults and neonates $(25,26)$. We found that the suppressive effect of PTX on phagocytosis was age- and dose-dependent. Monocytes and granulocytes from neonates were less influenced than were adult APCs. It has been demonstrated that IL-10 might enhance CD14-dependent phagocytosis (27). We therefore next explored the functional response to the ligand of TLR4/ CD14 (LPS) after PTX treatment. A dose-dependent downregulation of CD14 was found in all age groups, most pronounced in preterm neonates. Moreover, PTX led to a significant dose-dependent downregulation of TLR4 in monocytes of adults, and to a lesser degree in monocytes of preterm neonates. This effect was confirmed on mRNA level. The analysis of signal transduction in monocytes that had been pre-incubated with PTX before LPS stimulation showed a downregulation of phospho-NF- $\kappa B$ and phospho-ERK 1/2. These data go in line with the published results on decreased I $\kappa \mathrm{B}$ and NF- $\kappa \mathrm{B}$ nuclear translocation in adult PBMCs treated with PTX (19). TNF- $\alpha$ production is dependent on the activation of NF- $\mathrm{KB}$ genes, and downregulation of TNF- $\alpha$ 
a

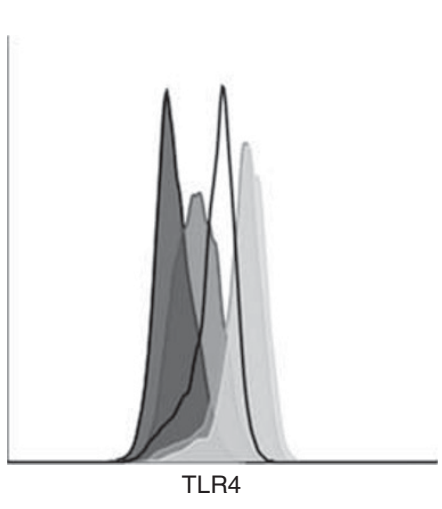

C

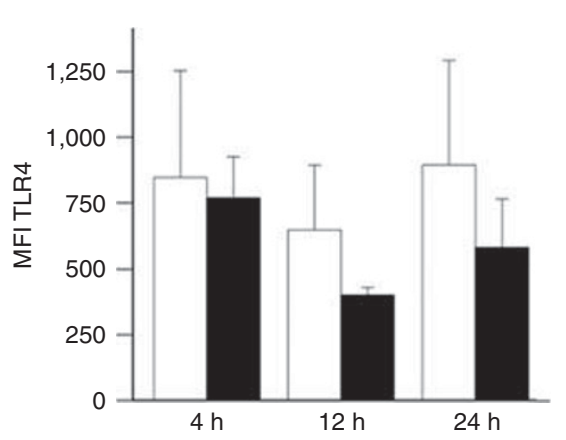

b

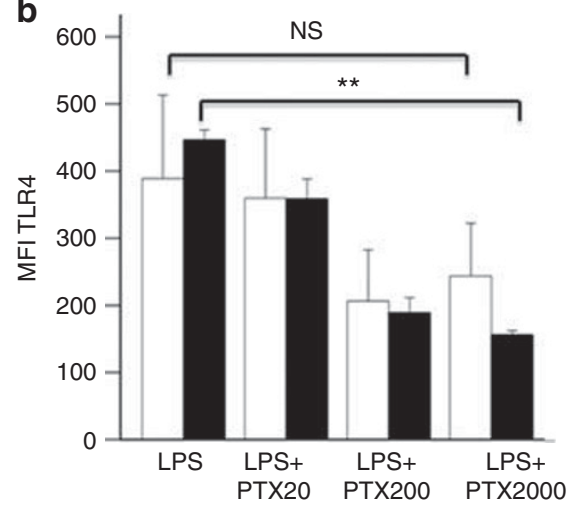

d

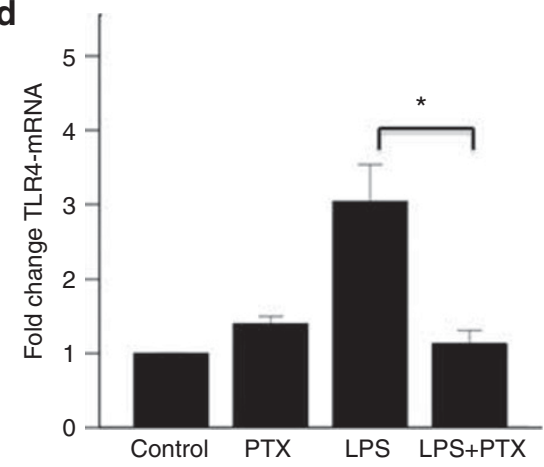

Figure 5. Effect of pentoxifylline (PTX) on Toll-like receptor 4 (TLR4) surface expression and TLR4 messenger RNA (mRNA) from monocytes of preterm neonates and adults. (a) Respective histograms of TLR4 expression on monocytes of a preterm neonate treated with LPS (10 ng/ml) and PTX $(20-2,000 \mu \mathrm{g} / \mathrm{ml})$. Black line with white filling indicates isotype, light gray indicates LPS only, medium gray indicates LPS + PTX $20 \mu \mathrm{g} / \mathrm{ml}$, dark gray indicates LPS+PTX $200 \mu \mathrm{g} / \mathrm{ml}$, and black fill indicates LPS+PTX $2000 \mu \mathrm{g} / \mathrm{ml}$. (b) Downregulation of TLR4 in monocytes of a whole-blood culture (24 h). In adults (filled boxes), TLR4 expression was significantly downregulated in a dose-dependent manner $(* * P<0.005)$. In preterm neonates (blank boxes), this effect was less pronounced and did not reach significance. Values are shown as mean fluorescence intensity (MFI). The experiment was conducted with three independent samples from adult and preterm infants each. Bars show mean value \pm SEM. (c) Downregulation of TLR4 in monocytes of a peripheral blood mononuclear cell culture incubated for $4-24 \mathrm{~h}$ with LPS ( $10 \mathrm{ng} / \mathrm{ml}$; blank boxes) and LPS+PTX (200 $\mu \mathrm{g} / \mathrm{ml}$; black boxes). Results indicate a downregulation of TLR4 starting at $12 \mathrm{~h}$ of PTX treatment, although not statistically significant. The experiment was conducted with three independent adult samples. Bars show mean value \pm SEM. (d) For TLR4 m-RNA, monocytes were stimulated with LPS (10 ng/ml) and PTX $(200 \mu \mathrm{g} / \mathrm{ml})$ for $6 \mathrm{~h}$. Reverse-transcriptase PCR was conducted as described in the Methods section, and m-RNA was normalized to 18s RNA. On mRNA level, TLR4 was upregulated upon LPS stimulation as expected. This effect was significantly suppressed if PTX was co-administrated $\left({ }^{*} P<0.05\right)$. PTX alone did not have any influence on TLR4 mRNA. Values are shown as fold change in relation to unstimulated control. The experiment was conducted with three independent adult samples.

production by IL-10 may occur through a mechanism involving the inhibition of $\mathrm{NF}-\kappa \mathrm{B}$ activation directly or indirectly by stabilizing $\mathrm{I} \kappa \mathrm{B}$ in human monocytes and macrophages $(28,29)$.

Age-dependent differences found in this study most likely arise from functional immunological immaturity of preterm neonates. Well-described differences between the adult and neonatal immune system concern physiological inhibitory substances in neonatal blood that might accelerate the effects of PTX. Neonatal blood plasma contains relatively high concentrations of adenosine, and neonatal cord blood MNCs demonstrate increased sensitivity to the cyclic AMP (cAMP)mediated inhibitory effects of adenosine (30). PTX is an inhibitor of PDEs and most of its functions are thought to be mediated by enhanced cyclic AMP levels as a result of reduced degradation of cyclic AMP via PDE (31). As shown for human PMN, immunomodulatory properties of PTX are mediated via adenosine-dependent pathways. Adenosine plus PTX lead to a profound inhibitory effect on pro-inflammatory cytokine production (32). It is conceivable that high adenosine concentrations in neonates contribute to a differentiated effect of PTX in the hyperinflammatory state of neonatal sepsis.

Further studies on (1) the mechanisms by which neonatal adenosine concentration influences the dose-dependent efficacy of PTX (2), the signaling capacities of TLR4 under the influence of PTX, and (3) the ability of PTX to reduce phagocytic activity are required to elucidate the complex mechanisms and interactions that may occur in the scenario of neonatal sepsis.

\section{Study Limitations}

The present study has several limitations. First, for some of our experiments, we used whole-blood assays to mimic the 
a

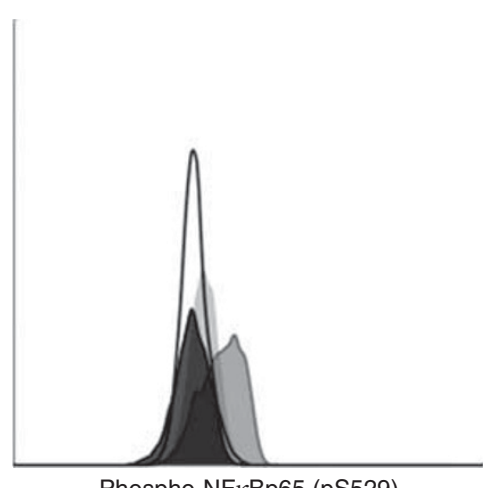

Phospho-NFkBp65 (pS529)

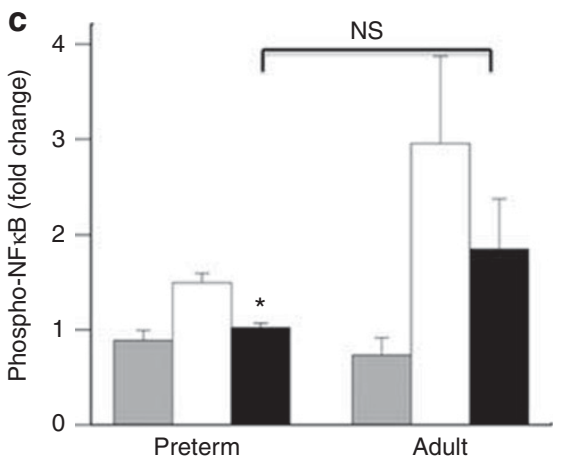

b

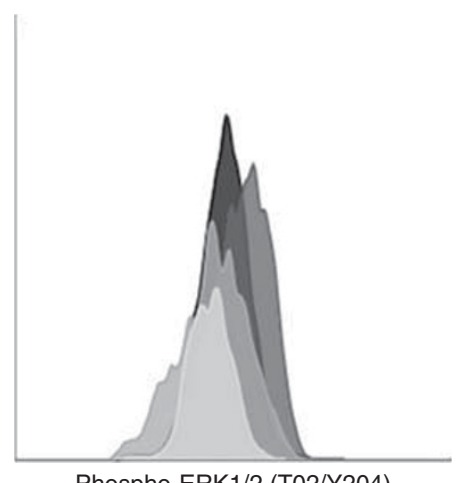

Phospho-ERK1/2 (T02/Y204)

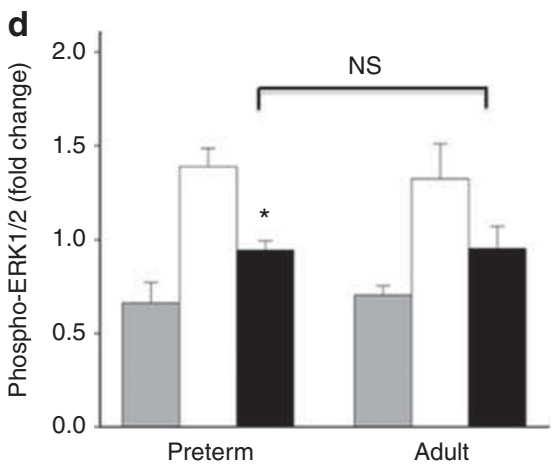

Figure 6. Effect of pentoxifylline (PTX) on phosphorylation pattern of nuclear factor-kB and ERK 1/2. Whole blood was incubated with PTX (200 $\mu \mathrm{g} /$ $\mathrm{ml}$ ) for $24 \mathrm{~h}$. Thereafter, blood was stimulated with LPS $(10 \mathrm{ng} / \mathrm{ml})$ for $10 \mathrm{~min}$ at $37^{\circ} \mathrm{C}$. (a) Respective histogram of phosphorylation of NF-KB. White filling indicates medium only, light gray indicates isotype, dark gray indicates LPS, and black indicates LPS+PTX. (b) Phosphorylation of ERK $1 / 2$ in CD14-positive monocytes of a preterm neonate. Light gray indicates medium only, medium gray indicates isotype, dark gray indicates LPS, and black indicates LPS+PTX. PTX stimulation resulted in a significant reduction of phospho-nuclear factor-KB (c) and phospho-ER 1/2 activity (d) in the preterm neonate $(* P<0.05)$. There was no age-dependent difference regarding this effect of PTX. Gray boxes indicate PTX, blank boxes indicate LPS, and black boxes indicate LPS+PTX. Mean data $( \pm$ SD) from adults and preterm neonates are shown.

physiological environment. However, by applying this method, our results might have been influenced by interference from other cells and mediators. For this reason, our main results (CD14, TLR4, and TNF- $\alpha$ ) were confirmed in monocytes from a PBMC culture or shown in primarily isolated monocytes (mRNA of TLR4). Because of the technical problems, we could not measure intracellular IL-10.

Second, incubation time was up to $24 \mathrm{~h}$ in the whole-blood culture in some experiments. We cannot exclude any influence of sample hypoxia or glucose deprivation on our results by the length of incubation time.

Third, because of the very small sample size volumes of cord blood in preterm neonates of $\leqslant 32$ weeks of gestation, not all tests could be performed with each sample of neonatal blood, and the number of individuals tested varied for each age group depending on the experiment.

\section{CONCLUSION}

The immunomodulatory properties of PTX cause a wide range of effects on differentiation, maturation, cytokine production, as well as TLR4 expression, signaling, and phagocytic activity of neonatal and adult monocytes. Our in vitro model provides insight into age-dependent differences of PTX with a focus on preterm infants. We found distinct immunological responses in preterm infants leading to quantitative and qualitative differences in the levels of surface markers and cytokine production. The present data contribute to our understanding of the therapeutic mechanisms and beneficial effects of PTX for adjunctive treatment of sepsis in preterm infants.

\section{ACKNOWLEDGMENTS}

The technical support by Günther Hofbauer is gratefully appreciated. We also thank Ludwig Gortner for corrections and Daniel Agustus for reading the manuscript.

\section{STATEMENT OF FINANCIAL SUPPORT}

The study was supported by a grant of the "Verein Unser Kind". No other external funding was provided.

Disclosure: The authors declare no conflict of interest.

\section{REFERENCES}

1. Stoll BJ, Hansen NI, Higgins RD, et al. Very low birth weight preterm infants with early onset neonatal sepsis: the predominance of gramnegative infections continues in the National Institute of Child Health and Human Development Neonatal Research Network, 2002-2003. Pediatr Infect Dis J 2005;24:635-9.

2. Chalmers IM, Janossy G, Contreras M, Navarrete C. Intracellular cytokine profile of cord and adult blood lymphocytes. Blood 1998;92:11-8. 
3. Sadeghi K, Berger A, Langgartner M, et al. Immaturity of infection control in preterm and term newborns is associated with impaired toll-like receptor signaling. J Infect Dis 2007;195:296-302.

4. Schüller SS, Sadeghi K, Wisgrill L, et al. Preterm neonates display altered plasmacytoid dendritic cell function and morphology. J Leukoc Biol 2013;93:781-8.

5. Levy O. Innate immunity of the newborn: basic mechanisms and clinical correlates. Nat Rev Immunol 2007;7:379-90.

6. Krakauer T, Oppenheim JJ. IL-1 and tumor necrosis factor-alpha each up-regulate both the expression of IFN-gamma receptors and enhance IFN-gamma-induced HLA-DR expression on human monocytes and a human monocytic cell line (THP-1). J Immunol 1993;150:1205-1.

7. Wisgrill L, Groschopf A, Herndl E, et al. Reduced TNF- $\alpha$ response in preterm neonates is associated with impaired nonclassic monocyte function. J Leukoc Biol 2016;100:607-12.

8. Förster-Waldl E, Sadeghi K, Tamandl D, et al. Monocyte toll-like receptor 4 expression and LPS-induced cytokine production increase during gestational aging. Pediatr Res 2005;58:121-4.

9. Lakhani SA, Bogue CW. Toll-like receptor signaling in sepsis. Curr Opin Pediatr 2003;15:278-82.

10. Shimaoka M, Park EJ. Advances in understanding sepsis. Eur J Anaesthesiol Suppl 2008;42:146-53.

11. Pammi M, Haque KN. Pentoxifylline for treatment of sepsis and necrotizing enterocolitis in neonates. Cochrane Database Syst Rev 2015;3: CD004205.

12. Shabaan AE, Nasef N, Shouman B, Nour I, Mesbah A, Abdel-Hady H. Pentoxifylline therapy for late-onset sepsis in preterm infants: a randomized controlled trial. Pediatr Infect Dis J 2015;34:e143-8.

13. Zabel P, Schade FU, Schlaak M. Inhibition of endogenous TNF formation by pentoxifylline. Immunobiology 1993;187:447-63.

14. D’Hellencourt CL, Diaw L, Cornillet P, Guenounou M. Differential regulation of TNF alpha, IL-1 beta, IL-6, IL-8, TNF beta, and IL-10 by pentoxifylline. Int J Immunopharmacol 1996;18:739-48.

15. Neuner P, Klosner G, Pourmojib M, Knobler R, Schwarz T. Pentoxifylline in vivo and in vitro down-regulates the expression of the intercellular adhesion molecule-1 in monocytes. Immunology 1997;90: $435-9$.

16. Wang W, Tam WF, Hughes CC, Rath S, Sen R. c-Rel is a target of pentoxifylline-mediated inhibition of T lymphocyte activation. Immunity 1997;6:165-74.

17. Szymura-Oleksiak J, Bury J, Lauterbach R, Pawłowski M. Serum Concentrations of pentoxifylline and its metabolites in premature infants with sepsis when administered by continuous intravenous infusion. Pharm Pharmacol Commun 1997;3:367-71.
18. Lauterbach R, Pawlik D, Kowalczyk D, Ksycínski W, Helwich E, Zembala M. Effect of the immunomodulating agent, pentoxifylline, in the treatment of sepsis in prematurely delivered infants: a placebocontrolled, double-blind trial. Crit Care Med 1999;27:807-14.

19. Coimbra R, Melbostad H, Loomis W, Tobar M, Hoyt DB. Phosphodiesterase inhibition decreases nuclear factor-kappaB activation and shifts the cytokine response toward anti-inflammatory activity in acute endotoxemia. J Trauma 2005;59:575-82.

20. Marcinkiewicz J, Grabowska A, Lauterbach R, Bobek M. Differential effects of pentoxifylline, a non-specific phosphodiesterase inhibitor, on the production of IL-10, IL-12 p40 and p35 subunits by murine peritoneal macrophages. Immunopharmacology 2000;49:335-43.

21. Rieckmann P, Weber F, Günther A, et al. Pentoxifylline, a phosphodiesterase inhibitor, induces immune deviation in patients with multiple sclerosis. J Neuroimmunol 1996;64:193-200.

22. Sanjabi S, Zenewicz LA, Kamanaka M, Flavell RA. Anti-inflammatory and pro-inflammatory roles of TGF-beta, IL-10, and IL-22 in immunity and autoimmunity. Curr Opin Pharmacol 2009;9:447-53.

23. Howard M, Muchamuel T, Andrade S, Menon S. Interleukin 10 protects mice from lethal endotoxemia. J Exp Med 1993;177:1205-8.

24. Howard M, O'Garra A, Ishida H, de Waal Malefyt R, de Vries J. Biological properties of interleukin 10. J Clin Immunol 1992;12:239-47.

25. Danikas DD, Karakantza M, Theodorou GL, Sakellaropoulos GC, Gogos CA. Prognostic value of phagocytic activity of neutrophils and monocytes in sepsis. Correlation to CD64 and CD14 antigen expression. Clin Exp Immunol 2008;154:87-97.

26. Falconer AE, Carr R, Edwards SW. Impaired neutrophil phagocytosis in preterm neonates: lack of correlation with expression of immunoglobulin or complement receptors. Biol Neonate 1995;68:264-9.

27. Lingnau M, Höflich C, Volk H-D, Sabat R, Döcke W-D. Interleukin-10 enhances the CD14-dependent phagocytosis of bacteria and apoptotic cells by human monocytes. Hum Immunol 2007;68:730-8.

28. Shames BD, Selzman CH, Meldrum DR, et al. Interleukin-10 stabilizes inhibitory kappaB-alpha in human monocytes. Shock 1998;10:389-94.

29. Ward PA, Lentsch AB. Endogenous regulation of the acute inflammatory response. Mol Cell Biochem 234-235:225-8.

30. Levy O, Coughlin M, Cronstein BN, Roy RM, Desai A, Wessels MR. The adenosine system selectively inhibits TLR-mediated TNF-alpha production in the human newborn. J Immunol 2006;177:1956-66.

31. Anaya JM, Espinoza LR. Phosphodiesterase inhibitor pentoxifylline: an antiinflammatory/immunomodulatory drug potentially useful in some rheumatic diseases. J Rheumatol 1995;22:595-9.

32. Kreth S, Ledderose C, Luchting B, Weis F, Thiel M. Immunomodulatory properties of pentoxifylline are mediated via adenosine-dependent pathways. Shock 2010;34:10-6. 